\title{
STUDY ON ROLE OF CORTICOCANCELLOUS GRAFT IN BONE DEFECTS CAUSED BY FRACTURES
}

\author{
G. Vara Prasad ${ }^{1}$ P. Anil Babu²
}

1 Professor, Department of Orthopaedics, Guntur Medical College, Guntur, Andhra Pradesh.

${ }^{2}$ Assistant Professor, Department of Orthopaedics, Guntur Medical College, Guntur, Andhra Pradesh.

ABSTRACT
BACKGROUND
Fractures often occur with irregular ends and bone loss to a variable extent. Bone graft has a well-recognised role in orthopaedic
surgery for the treatment of non-union, bridging diaphyseal and metaphyseal defects. The gap at the fracture site will definitely
hamper the fracture stability. Usually, the gap at the fracture site is more or less filled by fibrous tissue if not by the callus. The fate
of the fracture union depends on the gap filler at the same time not compromising with the stability.

\section{MATERIALS AND METHODS}

The purpose of this study is to investigate the role of bone grafts in aiding the fracture stability. Open reduction techniques often cause more fracture trimming resulting in non-reciprocating fracture surfaces that need a filler at the same time not compromising the stability. The harvested corticocancellous graft is fashioned to act like strut for 78 cases in our study.

\section{RESULTS}

The results are assessed by the approximation of the fracture ends and provisional stability given by the cortico-cancellous graft. Whatever graft that is harvested should serve the purpose not compromising either stability or gap filling.

\section{CONCLUSION}

Corticocancellous grafts serve the purpose of filling the fracture gaps as well as aiding in the stability of fracture when the fracture has created non-reciprocating ends at the fracture site.

\section{KEYWORDS}

Corticocancellous Bone Graft, Stability, Gap Filling.

HOW TO CITE THIS ARTICLE: Prasad GV, Babu PA. Study on role of corticocancellous graft in bone defects caused by fractures. J. Evolution Med. Dent. Sci. 2017;6(38):3114-3117, DOI: 10.14260/Jemds/2017/671

\section{BACKGROUND}

Bone grafts are obtained from different sources. The very purpose of graft should be to aid in the fracture union. Autogenous bone graft is osteogenic, osteoinductive, osteoconductive and completely biocompatible.

Osteogenic materials have the inherent capacity to form bone, which implies that they have living cells such as osteocytes or osteoblasts, capable of producing it. Osteoinductive materials stimulate cells in the wound or the local environment to undergo phenotypic conversion to osteoprogenitor cell types capable of formation of bone. Osteoconductive materials have no capability to form bone or induce its formation. They merely provide an inert biocompatible scaffold, which local osseous tissue can utilise to regenerate living bone.

There is no substitute for bone-graft yet available, which embodies all these qualities. To date most of the available materials have tended to be either predominantly osteogenic or osteoinductive or purely osteoconductive.(1) Bone defects in non-traumatic conditions, even before a fracture could occur are treated by bone grafts.(2)

Financial or Other, Competing Interest: None.

Submission 01-04-2017, Peer Review 01-05-2017,

Acceptance 06-05-2017, Published 11-05-2017.

Corresponding Author:

Dr. P. Anil Babu,

\#5-54-120, 101 Suryodaya Residency,

6/18, Brodipet,

Guntur-522002

Andhra Pradesh.

E-mail: anilpbabu@gmail.com

DOI: $10.14260 /$ jemds $/ 2017 / 671$
Bone defects may impede normal biomechanics and the structural stability of bone as an organ. In many cases, the correction of bone defects requires extensive surgical intervention involving the use of bone-grafting techniques and other procedures in which healing is slow, there is a high risk of infection and considerable pain is provoked with no guarantee of complete correction of the defect. Therefore, the search for surgical alternatives continues to present a major challenge in orthopaedic traumatology.(3) The most obvious alternative to autogenous bone graft has been allograft bone, which has been widely used in both structural and morsellised forms depending on the indication.

Allograft bone has been employed in fresh, frozen or freeze-dried forms. Fresh allografts are rarely used because of the immune response and the risk of transmission of disease. The frozen and freeze-dried types are osteoconductive, but are considered at best to be only weakly osteoinductive.

Allograft bone is not the usual choice in the management of orthopaedic trauma. In cases of inadequate autograft, the useful alternative is allograft. And also in elderly patients with osteoporosis in whom the availability of autografts of good quality is inadequate. Bulk allografts can be utilised for the treatment of segmental bone defects. Although, their use is well documented for reconstruction after resection of bone tumours, they are not commonly used in this way in reconstruction after trauma in which techniques of bone lengthening and transport are more usually employed.

Bone graft substitutes of two main types are now available. BMP in a carrier material, which after implantation induces local tissue to form bone. Osteoconductive materials are an inert scaffold, which allows bony ingrowth from local 
osseous tissue. Some of these products have compressive strengths similar to that of cancellous bone. Many are still being evaluated experimentally. There is increasing interest in combining an osteoinductive protein in an osteoconductive carrier medium with more desirable structural properties. Osteogenic proteins have met some success in the treatment of bone defects and non-union.

Autologous bone grafting will continue to play an important role in the treatment of non-union. Wherever the fracture fixation is done with non-reciprocating ends at fracture site, the graft material should be able to add to the stability till the union process utilise the scaffold to produce callus.

Lead is present in up to one-third of tibial allograft and autograft bone specimens at potentially osteotoxic levels regardless of the source or screening. Further research is needed to delineate the relationship with non-union or pseudoarthrosis after procedures in which allograft is used.(4) Anatomical plate combined with cortical bone plate allograft fixation is a good method to treat comminuted fractures of the femoral condyles. This method can effectively achieve complete cortical bone on the inside of the femur as well as provide rigid fixation. ${ }^{(5)}$

In response to the multifactorial origin of non-union, the Diamond Concept for treatment was developed and described by Giannoudis, Einhorn and Marsh and Giannoudis et al. The non-union is evaluated according to the following criteria: Osteogenic cells, osteoconductive scaffolds, mechanical environment, growth factors, vascularity.

By identifying the possible instability at fracture site, reasonable combination of biological and biomechanical approaches may greatly improve outcome. This study is intended to establish the role of bone graft as one of the stabiliser at the fracture site when the gap is due to nonreciprocating ends.

\section{MATERIALS AND METHODS}

Our study is an observational study on fracture gaps with doubtful mechanical stability, which consists of 78 cases of long bone fractures, wherein the non-reciprocating fracture ends are seen on pre-operative Radiographs. These fractures are prepared for open reduction and internal fixation. The pre-operative preparation includes fitness for surgery, in which open reduction and internal fixation is done for diaphyseal segments and corticocancellous junctions with bone grafting. Any kind of bone graft can basically fill the gaps caused by fractures; in our study, the graft is harvested wherein the cortical bone is carefully collected and nibbled to fill the gaps as well as give added stability at the fracture site. The surgeries are performed with pre- and intra-operative assessment to identify bone gaps that can be caused by nonreciprocating ends at the fracture site.

\section{Surgery and Intraoperative Findings}

All the cases are treated with open reduction and internal fixation. As the fracture site is exposed as per the preoperative findings, the fractures show comminution at the fracture site which often have loose fragments. As the fracture site is cleared off the loose fragments, which essentially lack soft tissue attachments, the gap presents variety of defects. These are unicortical defects, butterfly fragments, sclerotic ends of the bones. In this scenario, even the best devices will not give fracture reduction with good approximation of the ends. This kind of no approximation in spite of good reduction cannot be relied on to get union. We followed the principles mentioned above that the graft should be able to aid in giving mechanical strength to the reduction as well as filling the gaps.

For our study, the autologous bone grafts are harvested from the iliac crest as corticocancellous grafts, wherein the graft will have all the properties of an ideal graft.

The cancellous part of the graft will act as a gap filler and cortical part will aid in the stability at the fixation side. We found the fracture ends get approximated as reciprocating surfaces by the cortical portion of the graft. In the graft thus harvested is inserted in the defect, to span between the proximal and distal fragments from the medullary canal to medullary canal.

The sample size of this study is 78 cases. Within this the different types of fracture patterns are involved which are bound to show fracture gap and instability. The distribution of different patterns is Butterfly fragments 24, Oblique 31, Spiral 12 and Comminuted 21. Each fracture when reduced with fashioned corticocancellous bone graft is analysed to what extent the stability is achieved before plating, if the fracture is stable without reduction clamps it is graded as I. The gap at the fracture site if it is totally filled up with the graft is graded as I. This good quality of stability and gap closure is considered as favourable outcome in the study for the given fracture pattern.

\section{RESULTS}

The intraoperative assessment for stability is done by using bone hooks or bone holding forceps. The intraoperative stability is assessed during open reduction of the fractures, which have shown overriding of the fracture ends, oblique fracture patterns, partial cortical bone loss due to either trimming or loose fragments or splinters found intraoperatively; all of them are found to cause unstable reductions due to loss of bone. This assessment is done before and after bone gap. Before the bone graft is inserted the stability is checked at the fracture site. The abnormal mobility is checked whether it is causing opening at the fracture site or not. All the oblique fractures in our study are found to be unstable with fracture gap before grafting. Once bone graft is inserted into the medullary canal with proud graft from the fracture site, the reduction with angulation technique pushes the proud part into the other side of medullary canal and gives stability at the fracture site.

In fractures where much bone needs to be nibbled to create fresh edges the fractures are unstable at the same, sometimes they showed abnormal mobility before grafting. With the bone graft, the gaps caused by nibbled bone are closed, still the fracture shoed minimal abnormal mobility. Plating done for this minimal stability makes the graft totally incorporated into medullary canal, and thus closing the fracture gap.

All the fractures with butterfly fragments and oblique patterns showed total closure of the gap and stability.

The fractures with spiral pattern and comminution showed stability with $50 \%$ gap closure and $45 \%$ gap closure respectively. 


\begin{tabular}{|c|c|c|}
\hline Bone & Autologous Graft & Cases \\
\hline Femur & Iliac crest & 12 \\
\hline Tibia & Iliac crest & 18 \\
\hline Humerus & Iliac crest & 14 \\
\hline Radius & Iliac crest & 19 \\
\hline Ulna & Iliac crest & 15 \\
\hline \multicolumn{2}{|c|}{ Table 1. Fracture Distribution } \\
\hline
\end{tabular}

\begin{tabular}{|c|c|c|}
\hline Butterfly fragments & 24 & Stable, No Gap \\
\hline Oblique & 31 & Stable, No Gap \\
\hline Spiral & 12 & Stable, Min. Gap \\
\hline Comminuted & 11 & Stable, Min. Gap \\
\hline \multicolumn{3}{|c|}{ Table 2. Fracture Pattern } \\
\hline
\end{tabular}

\begin{tabular}{|c|c|c|c|c|}
\hline Pattern & Cases & Post Reduction & Total & Percentage of Efficacy \\
\hline Pattern & Stability & Gap & Stability 100\%, Gap closure: $100 \%$ \\
\hline Butterfly & I: 24 & I: 24 & 24 & Stability 100\%, Gap closure: $100 \%$ \\
\hline Oblique & I: 26 & I: 31 & 31 & Stability 100\%, Gap closure: $50 \%$ \\
\hline Spiral & I:12 & I:6 & 12 & Stability 100\%, Gap closure: $45 \%$ \\
\hline Comminuted & 1:11 & I:5 & 11 & Table 3. Analysis \\
\hline
\end{tabular}
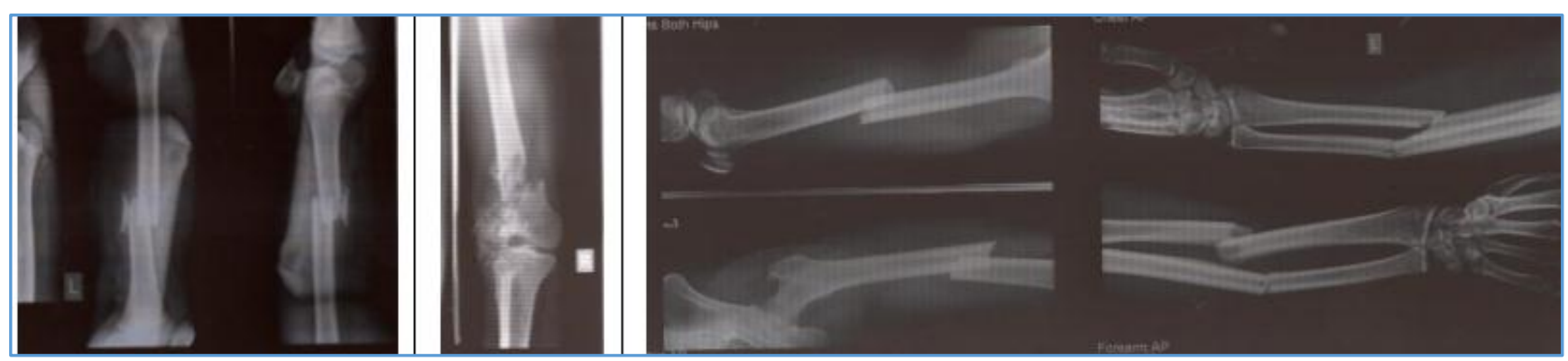

Figure 1. Pre-Operative
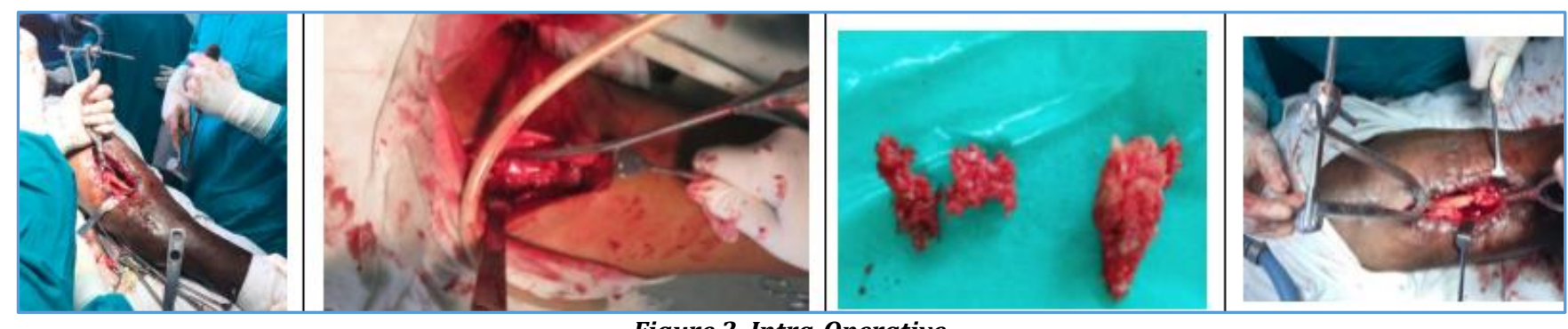

Figure 2. Intra-Operative
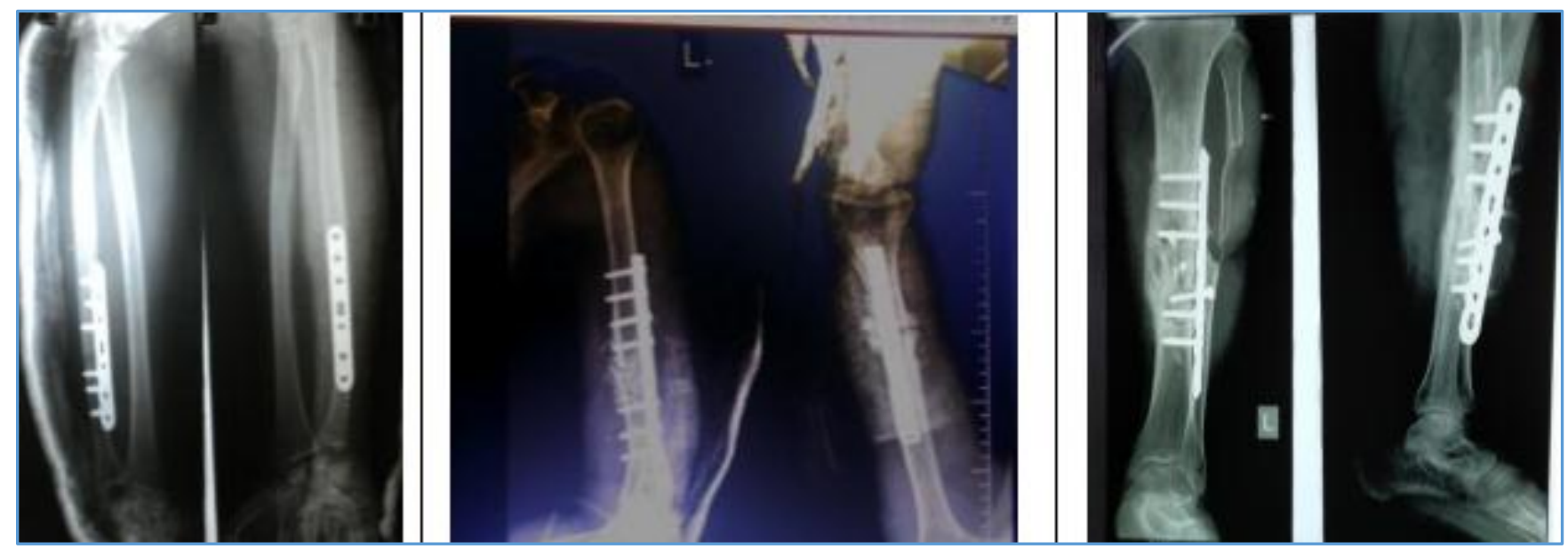

Figure 3. Post-Operative

\section{DISCUSSION}

Bone grafts which are impacted can readily serve the purpose of gap filling as well as restoring vascularity at fracture site. Fibular Allograft with Impaction Bone Grafting (FAIBG) is an effective head-preservation method for avoiding HR in the early stage of FHN. The advantage of this hip-preservation method is that it provides both repaired materials and biomechanical structural support during the healing of the necrosis region. ${ }^{(6)}$

Fracture gaps which are seen on radiographs and intraoperatively require fixation to give stability as well as closure of the gap. When creating a freshened edge of the fracture it 
self-causes a defect; the graft should be able to fill the defect and also give the stability during the manipulation and fixation. Cancellous graft alone may fill the gap, but when the fracture needs stability especially in un-reciprocating ends corticocancellous grafts serve both the purposes. The graft which is fashioned to have both cancellous and cortical components can be firmly placed between the fracture ends, even when there is no close approximation. The fracture patterns, which show instability are better treated with strut like grafts for stability and gap closure.

The graft that retains all the ideal properties should be chosen while fixing a fracture with rigid fixation as well as the restoration of blood supply.

\section{CONCLUSION}

Fracture gaps which are seen on radiographs and intraoperatively require fixation to give stability as well as closure of the gap. When creating a freshened edge of the fracture itself causes a defect; the graft should be able to fill the defect and also give the stability during the manipulation and fixation. Cancellous graft alone may fill the gap, but when the fracture needs stability, especially in non-reciprocating ends, corticocancellous grafts serve both the purposes. The graft which is fashioned to have both cancellous and cortical components can be firmly placed between the fracture ends, even when there is no close approximation. The fracture patterns, which show instability are better treated with strutlike grafts for stability and gap closure.

\section{REFERENCES}

[1] Keating JF, McQueen MM. Substitutes for autologous bone graft in orthopaedic trauma. J Bone Joint Surg Br 2001;83(1):3-8.

[2] Majoor BCJ, Peeters-Boef MJM, van de Sande MAJ, et al. What is the role of allogeneic cortical strut grafts in the treatment of fibrous dysplasia of the proximal femur? Clin Orthop Relat Res 2017;475(3):786-95.

[3] Rankine JJ, Hodgson RJ, Tan HB, et al. MRI appearances of the femur following bone graft harvesting using the Reamer Irrigator Aspirator. Injury 2015;(46 Suppl 8):S65-7.

[4] Behrend C, Carmouche J, Millhouse PW, et al. Allogeneic and autogenous bone grafts are affected by historical donor environmental exposure. Clin Orthop Relat Res 2016;474(6):1405-9.

[5] Guo Z, Gong X, Li Y, et al. Anatomical plate combined with cortical bone plate allografts for treatment of comminuted fractures of femoral condyles. Zhongguo Xiu Fu Chong Jian Wai Ke Za Zhi 2015 Jan;29(1):6-9.

[6] Zhou G, Zhang Y, Zeng L, et al. Should thorough debridement be used in fibular allograft with impaction bone grafting to treat femoral head necrosis: a biomechanical evaluation. BMC Musculoskeletal Disorders 2015;16:140. 